\title{
Isolation, Characterization and Antagonistic Activity of Fluorescent Pseudomonads
}

\author{
Priyanka $^{1 *}$, Geeta Goudar ${ }^{2}$, P. Jones Nirmal Nath ${ }^{1}$ and P.V. Patil ${ }^{3}$ \\ ${ }^{1}$ Department of Agriculture Microbiology, AC, Dharwad, UAS, Dharwad, Karnataka, India \\ ${ }^{2}$ Department of Agriculture Microbiology, AC, Vijayapura, UAS, Dharwad, Karnataka, India \\ ${ }^{3}$ Department of Plant Pathology, AC, Dharwad, UAS, Dharwad, Karnataka, India \\ *Corresponding author
}

\section{A B S T R A C T}

\begin{tabular}{l} 
K e y w o r d s \\
$\begin{array}{l}\text { Fluorescent } \\
\text { pseudomonads, } \\
\text { Sclerotium rolfsii, } \\
\text { Colletotricum } \\
\text { truncatum, PGPR. }\end{array}$ \\
\hline Article Info \\
$\begin{array}{l}\text { Accepted: } \\
28 \text { October } 2017 \\
\text { Available Online: } \\
10 \text { December } 2017\end{array}$ \\
\hline
\end{tabular}

An attempt was made to isolate 62 strains of fluorescent pseudomonads from rhizosphere of soybean collected from two soybean growing areas viz., Belagavi and Dharwad districts of Karnataka. These isolates were subjected for morphological, biochemical and functional characterization. All the isolates were gram negative rods. Colour of the colony varied from light green, green, dark green to light orange. The isolates showed fluorescens under UV light. Out of 62 isolates 36, 24, 52, 36, 39 isolates were positive for starch hydrolysis, arginine hydrolysis, oxidase test, denitrification and gelatin liquification respectively. These isolates exhibited wide variations for their ability to utilize different carbon sources. With respect to MPS, 52 isolates showed MPS activity. Forty seven isolates were positive for $\mathrm{HCN}$ production, in which 10 of the isolates were strong $\mathrm{HCN}$ producers. All the isolates were positive for siderophore production, which ranged between 6.00 to $36.37 \mathrm{~mm}$. Whereas, IAA and GA production ranged from 39 to 28.03 and 0.5 to $18.52 \mu \mathrm{g} / 25 \mathrm{ml}$ of broth respectively. All the 62 isolates were assayed for their antagonistic activity against Sclerotium rolfsii and Colletotricum truncatum using dual plate technique. Out of 62 isolates, 51 and 38 were inhibitory to $S$. rolfsii and $C$. truncatum respectively.

\section{Introduction}

Rhizobacteria that exert beneficial effects on plant growth and development are referred to as plant growth promoting rhizobacteria (PGPR) (Ashrafuzzaman et al., 2009). PGPR promote plant growth by various factors like ability to produce plant growth regulators, asymbiotic $\mathrm{N}_{2}$ fixation, antagonism against phytopathogenic microorganisms by production of siderophores, antibiotics and cyanide, solubilization of mineral phosphates and other nutrients (Sarvanakumar et al., 2007). PGPR may use more than one of above mechanisms to enhance plant growth as experimental evidence suggests that the plant growth stimulation is the net result of multiple mechanisms that may be activated simultaneously (Martinez et al., 2010). Pseudomonas is diverse genus that occupies many different niches and exhibits versatile metabolic capacity (Clarke, 1982).

Fluorescent pseudomonads (FP's) a group of PGPR, have frequently been considered as effective biological control agents against soil-borne plant pathogens due to their rapid and aggressive colonization of plant roots. 
They produce secondary metabolites with antibiotic properties such as phytohormones, volatile compounds, hydrogen cyanide $(\mathrm{HCN})$ and siderophores. Plant growth-promoting ability of these bacteria is mainly due to the production of indole-3-acetic acid (IAA), siderophores and antibiotics (Lautenberg et al., 2001). Such strains can protect plants from various soil borne pathogens and/or stimulate plant growth (Haas and Defago, 2005). The abundance of literature on genus Pseudomonas is due to their elevated metabolic versatility capable of utilizing a wide range of simple and complex organic compounds and holding an important position in biosphere ecology (Scarpellini et al., 2004).

Antagonistic activity of Pseudomonas fluorescens and Pseudomonas putida in the rhizosphere has been recognized as major factor in the suppression of many phytopathogens. Bacteria of the genus Pseudomonas comprise a large group of the active biocontrol strains as a result of their general ability to produce a diverse array of potent antifungal metabolites.

Strains of Pseudomonas fluorescens are known to show biological control activity against certain soil-borne phytopathogenic fungi viz., Macrophomina phaseolina, Rhizoctonia solani, Phytophthora nicotianae var. parasitica, Pythium sp. and Fusarium sp. (Anand et al., 2010).

\section{Materials and Methods}

\section{Isolation of fluorescent pseudomonads}

A total of 37 soybean rhizosphere soils were collected from major soybean growing areas of Dharwad (15) and Belagavi (22) districts of Karnataka at crop maturity stage and used for the isolation of fluorescent pseudomonads on King's B medium using serial dilution and spread plate technique.

\section{Characterization of FP isolates}

The colonies showing greenish to yellowish pigmentation on King's B medium were picked up and observed for fluorescence under UV light. The isolates were also studied for their colony morphology, cell shape and gram reaction as per the standard procedure given by Barthalomew and Mittewar (1950) and Anonymous (1957). The isolates were subjected to biochemical characterization by employing the standard procedures given by Cappuccino and Sherman (1992). Different biochemical tests performed were denitrification, starch hydrolysis, arginine hydrolysis, gelatin liquification and Oxidase test.

\section{Functional Characterization of FP isolates}

The isolates were tested for their ability to solubilise $\mathrm{P}, \mathrm{HCN}$ production, Siderophore production and ability to produce plant growth promoting substances like IAA and GA.

In vitro screening of fluorescent pseudomonad isolates against fungal pathogens of soybean

All the 62 FP isolates were studied for their antagonistic activity against two fungal pathogens viz., Sclerotium rolfsii Sacc., causing collor or root rot (soil borne) and Colletotricum truncatum causing anthracnose (foliar pathogen) in soybean plant under in vitro condition, these pathogens also causes variety of diseases in many crops.

The pure cultures of S.rolfsii and $C$. truncatum were obtained from the Department of Plant Pathology, University of Agricultural Sciences, Dharwad. The dual inoculation technique of Sakthivel and Gnanamanickam (1987) were followed to study the antagonistic activity of the 
fluorescent pseudomonads against soil borne and foliar plant pathogens.

\section{Results and Discussion}

Isolation of fluorescent pseudomonads from rhizosphere soil was carried out by employing serial dilution and plating on King's B selective media. It may be attributed to specific choice of media employed for isolation viz., Nutrient agar and King's B. Several others hence supported to use King's B medium for isolation of fluorescent Pseudomonas sp. (King et al., 1954). For the isolation of pseudomonads and fluorescent Pseudomonas sp., TSA and King's B agar medium were also used (Raaijmakers and Weller, 1998). Total of 62 FP isolates were obtained from the 37 rhizosphere soil samples collected from the major soybean growing areas of Dharwad and Belagavi district of Karnataka (Table 1).

All 62 isolates were used to study morphological traits. The colony morphology of isolates was found to be round to irregular shape and the irregular shaped colonies were found to be spreading type. All the 62 isolates exhibited fluorescence under UV light. However variations with respect to intensity of fluorescence and colony morphology were observed. Based on intensity of fluorescence under UV light, the isolates were classified as very good fluorescence $(+++)$, medium fluorescence $(++)$ and low fluorescence $(+)$. Out of 62 isolates, 27 showed very good fluorescence $(+++)$ and 32 isolated showed medium fluorescence (++) under UV light.

Fluorescence is an important trait for identification, characterization and grouping of fluorescent pseudomonads (Brown and Lowbury, 1968). Among the 62 isolates majority of the isolates produced light green to green pigmented colonies, while some of the isolates appeared to have yellowish orange colonies. Colonies showing fluorescence under transilluminator were marked and a loopful of growth was picked in the laminar air flow for further purification. This could be a useful practice to obtain fluorescent pseudomonads from plate containing several isolated colonies from soil or root samples. It is reported from previous studies that some pigments such as carotenoids produced by pseudomonads species do not diffuse in to the medium and such colonies were found to have yellowish green colour which could resemble other fluorescing pigments (Indi, 2010). The cell morphological studies and the Gram reaction test revealed that all the 62 isolates were rod shaped and displayed a negative reaction for the Gram staining.

The biochemical characterization indicated that out of 62 isolates, 36 were positive for starch hydrolysis and 24 for arginine hydrolysis, 52 for oxidase test, 39 were positive for gelatin liquefaction and 36 exhibited the denitrification ability (Table 2). These results are in conformity with the work of Singh et al., (2007), who reported that out of seventeen isolates, seven isolates were identified to be Pseudomonas sp. on the basis of their cultural, morphological and biochemical characters. Similarly, Thirty five isolates of Pseudomonas fluorescens were isolated from the rhizosphere of rice fields by Meera and Balabaskar (2012). Among these, seven isolates which showed bright fluorescence under UV light were confirmed to be $P$. fluorescens after cultural and biochemical studies.

All the 62 isolates were also exercised to study the ability of the isolates to grow at $4^{0} \mathrm{C}$ and $41^{\circ} \mathrm{C}$ temperature. It was observed that there is an existence of variation among the isolates. Fifty two of the isolates were able to grow at $4{ }^{0} \mathrm{C}$ temperature and 10 isolates showed growth at $41{ }^{0} \mathrm{C}$ (Table 3 ). The 
findings are in conformity with the work of Suman et al., (2016) who revealed that, among nineteen isolates, eight isolates showed growth at $4{ }^{\circ} \mathrm{C}$ and eleven isolates at $42{ }^{0} \mathrm{C}$.

The isolates were also tested for their ability to utilize different carbon sources viz., glucose, lactose, sucrose, xylose, ribose, $\beta$ alanine, meso-inositol, mannitol, maltose, trehalose, L- valine and geraniol. The results revealed that among 62 isolates, 60, 56, 28, $29,35,52,52,25,54,52,19$ and 35 isolates were able to utilize glucose, lactose, sucrose, xylose, ribose, $\beta$-alanine, meso-inositol, mannitol, maltose, trehalose, L- valine and geraniol carbon sources respectively. None of the isolates showed growth on rhamnose (Table 3).

By considering the morphological, biochemical characteristics and ability of the isolates to grow at $4{ }^{\circ} \mathrm{C}$ and $41^{\circ} \mathrm{C}$ and utilize different carbon sources for their growth, 50 isolates were tentatively identified as $P$. fluorescens, 10 as $P$. aeruginosa and 2 isolates as $P$. aureofaciens strains based on Bergey's manual of Bacteriology. The results pertaining to characterization in this study are in line with the observation made by Paramageetham and Prasada Babu, 2012; Suman et al., 2016.

All the isolates were also examined for their functional properties like, P-solubilization, production of plant growth promoting substances and their biocontrol potential (Table 4). Out of 62 isolates, 52 were able to produce clear zone of $\mathrm{P}$ - solubilization (TCP) on Pikovskaya's agar medium. These isolates displayed wide variations in the diameter of the zone of solubilization, which varied from $5.31-21.71 \mathrm{~mm}$. The extent of zone of solubilization may or may not correlate with the amount of $\mathrm{P}$ solubilized (Rashid et al., 2004). Isolates of Pseudomonas fluorescens species differ in the ability to produce phosphatase enzyme and production of organic acids and hence showed different solubilization efficiency.

Fluorescent pseudomonads offer an interesting biological system with their ability to promote plant growth directly through production of plant growth promoting substances (IAA and GA) and indirectly through control of plant pathogens and deleterious organisms or both (Bakthavatchalu et al., 2012).

Seed bacterization with such organisms has emerged as a powerful technology to enhance plant growth and yield, besides providing protection against diseases. Earlier, Suneesh (2004) and Megha et al., (2007a) made an attempt to characterize PGPR isolates of Western Ghats and studied their functional diversity. Their efforts helped in identifying several PGPR with novel traits useful in agriculture. The present study is complimented with the previous work done. All 62 isolates were screened for their ability to produce IAA and GA. All the fluorescent pseudomonads in the present study produced significantly varying quantities of IAA (3.90 $\mu \mathrm{g}$ to $28.89 \mu \mathrm{g}$ IAA/25 ml of broth) (Table 4). Among the isolates, DFP48 recorded highest IAA production of $28.89 \mu \mathrm{g} / 25 \mathrm{ml}$.

Isolates from the rhizosphere are more efficient auxin producers than isolates from the bulk soil (Sarwar and Kremer, 1992) and IAA production by PGPR can vary among different species and strains, and it is also influenced by culture condition, growth stage and substrate availability (Mirza et al., 2001). The results obtained in this study are in line with the observation made by Khakipour et al., (2008), who reported that the IAA produced by $P$. fluorescens and $P$. putida strains varied from 0 to $31.6 \mathrm{mg} / \mathrm{l}$ and 0 to $24.08 \mathrm{mg} / \mathrm{l}$, respectively. 
Table.1 Morphological characterization of native fluorescent pseudomonad isolates

\begin{tabular}{|c|c|c|c|c|c|}
\hline Sl. NO & Isolate code & Colony morphology & Pigmentation (Under UV light) & Cell shape & Gram Reaction \\
\hline 1 & BFP1 & Round & Dull green & Rod & Gram-ve \\
\hline 2 & BFP2 & Irregular & Dull green & Rod & Gram -ve \\
\hline 3 & BFP3 & Irregular & Dull green & Rod & Gram -ve \\
\hline 4 & BFP4 & Irregular & Dull green & Rod & Gram -ve \\
\hline 5 & BFP5 & Round & Dull green & Rod & Gram-ve \\
\hline 6 & BFP6 & Round & Dull green & Rod & Gram -ve \\
\hline 7 & BFP7 & Irregular & Yellowish orange & Rod & Gram -ve \\
\hline 8 & BFP8 & Irregular & Yellowish orange & Rod & Gram -ve \\
\hline 9 & BFP9 & Round & Dark green & Rod & Gram -ve \\
\hline 10 & BFP10 & Round & Dark green & Rod & Gram -ve \\
\hline 11 & BFP11 & Round & Green & Rod & Gram -ve \\
\hline 12 & BFP12 & Round & Green & Rod & Gram -ve \\
\hline 13 & BFP13 & Irregular & Light orange & Rod & Gram -ve \\
\hline 14 & BFP14 & Irregular & Light orange & Rod & Gram -ve \\
\hline 15 & BFP15 & Round & Light orange & Rod & Gram -ve \\
\hline 16 & BFP16 & Irregular & Green & Rod & Gram -ve \\
\hline 17 & BFP17 & Irregular & Green & Rod & Gram -ve \\
\hline 18 & BFP18 & Round & Green & Rod & Gram -ve \\
\hline 19 & BFP19 & Round & Dull orange & Rod & Gram -ve \\
\hline 20 & BFP20 & Round & Dull orange & Rod & Gram -ve \\
\hline 21 & BFP21 & Round & Dull green & Rod & Gram -ve \\
\hline 22 & BFP22 & Round & Yellowish orange & Rod & Gram -ve \\
\hline 23 & BFP23 & Round & Dull green & Rod & Gram -ve \\
\hline 24 & BFP24 & Round & Dull green & Rod & Gram -ve \\
\hline 25 & BFP25 & Round & Green & Rod & Gram -ve \\
\hline 26 & BFP26 & Irregular & Dull green & Rod & Gram -ve \\
\hline 27 & BFP27 & Irregular & Dull green & Rod & Gram -ve \\
\hline 28 & BFP28 & Irregular & Dull green & Rod & Gram -ve \\
\hline 29 & BFP29 & Irregular & Dull green & Rod & Gram -ve \\
\hline 30 & BFP30 & Round & Dull green & Rod & Gram -ve \\
\hline 31 & BFP31 & Round & Dull green & Rod & Gram -ve \\
\hline 32 & BFP32 & Round & Dull green & Rod & Gram -ve \\
\hline 33 & BFP33 & Round & Dull green & Rod & Gram -ve \\
\hline 34 & BFP34 & Irregular & Dull green & Rod & Gram-ve \\
\hline 35 & BFP35 & Irregular & Dull green & Rod & Gram-ve \\
\hline 36 & BFP36 & Irregular & Dull green & Rod & Gram-ve \\
\hline 37 & BFP37 & Round & Green & Rod & Gram-ve \\
\hline 38 & BFP38 & Irregular & Green & Rod & Gram -ve \\
\hline 39 & BFP39 & Irregular & Dull green & Rod & Gram-ve \\
\hline 40 & BFP40 & Irregular & Yellowish orange & Rod & Gram-ve \\
\hline 41 & BFP41 & Round & Yellowish orange & Rod & Gram-ve \\
\hline 42 & BFP42 & Round & Dark green & Rod & Gram-ve \\
\hline 43 & BFP43 & Irregular & Dull green & Rod & Gram-ve \\
\hline 44 & BFP44 & Irregular & Slight orange & Rod & Gram-ve \\
\hline 45 & BFP45 & Irregular & Slight orange & Rod & Gram-ve \\
\hline 46 & DFP46 & Round & Green & Rod & Gram-ve \\
\hline 47 & DFP47 & Round & Green & Rod & Gram-ve \\
\hline 48 & DFP48 & Round & Yellowish orange & Rod & Gram-ve \\
\hline 49 & DFP49 & Round & Yellowish orange & Rod & Gram-ve \\
\hline 50 & DFP50 & Round & Dull green & Rod & Gram-ve \\
\hline 51 & DFP51 & Round & Dull green & Rod & Gram-ve \\
\hline 52 & DFP52 & Irregular & Dull green & Rod & Gram-ve \\
\hline 53 & DFP53 & Irregular & Green & Rod & Gram-ve \\
\hline 54 & DFP54 & Irregular & Green & Rod & Gram-ve \\
\hline 55 & DFP55 & Irregular & Green & Rod & Gram-ve \\
\hline 56 & DFP56 & Round & Yellowish orange & Rod & Gram-ve \\
\hline 57 & DFP57 & Round & Yellowish orange & Rod & Gram -ve \\
\hline 58 & DFP58 & Irregular & Dull green & Rod & Gram-ve \\
\hline 59 & DFP59 & Irregular & Dull green & Rod & Gram-ve \\
\hline 60 & DFP60 & Irregular & Dull green & Rod & Gram-ve \\
\hline 61 & DFP61 & Irregular & Yellowish orange & Rod & Gram -ve \\
\hline 62 & DFP62 & Round & Green & Rod & Gram -ve \\
\hline
\end{tabular}


Table.2 Biochemical characterization of native fluorescent pseudomonads

\begin{tabular}{|c|c|c|c|c|c|c|c|}
\hline SI.NO & Isolates & Denitrification & Starch hydrolysis & Arginine hydrolysis & Gelatin liquification & Oxidase test & Tentaive Identification \\
\hline 1 & BFP1 & - & - & - & + & + & P. fluorescens \\
\hline 2 & BFP2 & - & + & - & + & + & P. fluorescens \\
\hline 3 & BFP3 & - & + & - & - & + & Pseudomomas spp \\
\hline 4 & BFP4 & - & - & - & - & + & P. fluorescens \\
\hline 5 & BFP5 & - & + & - & - & + & P. aeruginosa \\
\hline 6 & BFP6 & - & + & + & - & - & Pseudomomas spp \\
\hline 7 & BFP7 & - & + & - & + & + & P. fluorescens \\
\hline 8 & BFP8 & + & - & - & - & + & P. fluorescens \\
\hline 9 & BFP9 & + & - & - & - & + & P. fluorescens \\
\hline 10 & BFP10 & - & - & - & + & + & P. fluorescens \\
\hline 11 & BFP11 & - & + & + & + & + & P. fluorescens \\
\hline 12 & BFP12 & - & + & - & + & + & P. fluorescens \\
\hline 13 & BFP13 & + & - & - & + & + & P. fluorescens \\
\hline 14 & BFP14 & + & - & - & - & + & P. fluorescens \\
\hline 15 & BFP15 & - & + & + & + & + & P. fluorescens \\
\hline 16 & BFP16 & - & + & - & + & + & P. fluorescens \\
\hline 17 & BFP17 & + & - & + & - & + & P. fluorescens \\
\hline 18 & BFP18 & - & - & - & + & - & P. aeruginosa \\
\hline 19 & BFP19 & + & - & - & - & + & P. fluorescens \\
\hline 20 & BFP20 & - & + & - & + & + & P. fluorescens \\
\hline 21 & BFP21 & + & + & + & - & - & P. aeruginosa \\
\hline 22 & BFP22 & - & - & - & + & - & P. fluorescens \\
\hline 23 & BFP23 & - & - & + & - & - & P. aeruginosa \\
\hline 24 & BFP24 & - & - & - & - & + & P. aeruginosa \\
\hline 25 & BFP25 & - & - & - & - & + & P. fluorescens \\
\hline 26 & BFP26 & - & - & - & + & - & P. aeruginosa \\
\hline 27 & BFP27 & - & + & - & + & + & P. fluorescens \\
\hline 28 & BFP28 & + & + & - & - & + & P. fluorescens \\
\hline 29 & BFP29 & + & - & - & - & + & P. fluorescens \\
\hline 30 & BFP30 & + & + & - & + & - & P. aeruginosa \\
\hline 31 & BFP31 & + & + & + & + & + & P. fluorescens \\
\hline 32 & BFP32 & - & + & + & + & + & P. fluorescens \\
\hline 33 & BFP33 & + & + & + & + & + & P. fluorescens \\
\hline 34 & BFP34 & + & + & - & - & + & P. fluorescens \\
\hline 35 & BFP35 & + & + & - & - & + & P. fluorescens \\
\hline 36 & BFP36 & + & + & + & + & + & P. fluorescens \\
\hline 37 & BFP37 & + & + & + & - & + & P. fluorescens \\
\hline 38 & BFP38 & + & + & + & + & + & P. fluorescens \\
\hline 39 & BFP39 & - & + & + & - & + & P. fluorescens \\
\hline 40 & BFP40 & + & + & + & + & + & P. fluorescens \\
\hline 41 & BFP41 & + & + & - & + & - & P. aeruginosa \\
\hline 42 & BFP42 & + & + & - & - & + & P. fluorescens \\
\hline 43 & BFP43 & + & + & + & - & + & P. fluorescens \\
\hline 44 & BFP44 & + & + & - & + & + & P. fluorescens \\
\hline 45 & DFP45 & + & + & + & + & + & P. fluorescens \\
\hline 46 & DFP46 & + & + & + & + & + & P. fluorescens \\
\hline 47 & DFP47 & + & - & - & + & + & P. fluorescens \\
\hline 48 & DFP48 & + & - & - & - & + & P. fluorescens \\
\hline 49 & DFP49 & - & + & + & + & + & P. fluorescens \\
\hline 50 & DFP50 & + & - & - & + & + & P. aeruginosa \\
\hline 51 & DFP51 & + & - & + & + & + & P. fluorescens \\
\hline 52 & DFP52 & + & - & - & + & + & P. fluorescens \\
\hline 53 & DFP53 & - & - & - & - & + & P. fluorescens \\
\hline 54 & DFP54 & + & - & - & - & - & P. fluorescens \\
\hline 55 & DFP55 & + & - & - & + & + & P. fluorescens \\
\hline 56 & DFP56 & + & + & - & + & + & P. fluorescens \\
\hline 57 & DFP57 & + & + & + & + & - & P. aeruginosa \\
\hline 58 & DFP58 & + & - & + & + & + & P. fluorescens \\
\hline 59 & DFP59 & - & + & - & + & + & P. fluorescens \\
\hline 60 & DFP60 & + & + & + & + & + & P. fluorescens \\
\hline 61 & DFP61 & + & + & + & + & + & P. fluorescens \\
\hline 62 & DFP62 & + & + & + & + & + & P. fluorescens \\
\hline
\end{tabular}

Note: (+) positive reaction for the test, (-) negative reaction for the test 
Table.3 Ability of fluorescent pseudomonad isolates to utilize different carbon sources and their ability to grow at $4{ }^{0} \mathrm{C}$ and $41{ }^{0} \mathrm{C}$ temperature

\begin{tabular}{|c|c|c|c|c|c|c|c|c|c|c|c|c|c|c|c|c|}
\hline \multicolumn{15}{|c|}{ Ability of fluorescent pseudomonad isolates to utilize different carbon sources } & \multicolumn{2}{|c|}{ Growth at } \\
\hline $\begin{array}{l}\text { Sl. } \\
\text { No }\end{array}$ & Isolates & Glucose & Lactose & Sucrose & Xylose & Ribose & B-alanine & Rhamose & $\begin{array}{l}\text { Meso- } \\
\text { Inositol }\end{array}$ & Mannitol & Maltose & Trehalose & L-valine & Geraniol & $4^{0} \mathrm{C}$ & $\begin{array}{r}41 \\
{ }^{0} \mathrm{C} \\
\end{array}$ \\
\hline & & & & & & & & & & & & & & & + & - \\
\hline 2 & BFP2 & + & - & - & - & - & + & - & - & - & - & - & - & - & + & - \\
\hline 3 & BFP3 & + & + & - & - & + & + & - & - & - & + & + & - & + & + & - \\
\hline 4 & BFP4 & + & - & - & - & + & + & - & - & - & - & + & - & + & + & - \\
\hline 5 & BFP5 & + & + & - & - & - & + & - & + & - & + & + & - & - & + & - \\
\hline 6 & BFP6 & + & + & - & - & + & + & - & + & - & + & - & - & + & - & + \\
\hline 7 & BFP7 & + & + & - & - & + & + & - & + & - & + & - & - & + & + & - \\
\hline 8 & BFP8 & + & + & + & + & + & + & - & + & + & + & + & + & + & + & - \\
\hline 9 & BFP9 & + & + & - & + & + & + & - & - & - & + & + & - & + & + & - \\
\hline 10 & BFP10 & + & + & - & - & - & + & - & - & - & + & + & - & - & + & - \\
\hline 11 & BFP11 & + & + & - & - & - & + & - & - & - & + & + & - & - & + & - \\
\hline 12 & BFP12 & + & + & - & - & - & + & - & + & - & - & + & - & - & + & - \\
\hline 13 & BFP13 & + & + & - & - & - & + & - & + & - & + & + & - & - & + & - \\
\hline 14 & FBP14 & + & - & - & - & - & + & - & + & - & + & + & - & - & + & - \\
\hline 15 & BFP15 & + & + & - & - & - & + & - & + & - & + & + & - & - & + & - \\
\hline 16 & BFP16 & + & + & - & - & + & + & - & + & - & + & + & - & + & + & - \\
\hline 17 & BFP17 & + & + & - & - & - & + & - & + & - & + & + & - & - & + & - \\
\hline 18 & BFP18 & + & + & - & - & - & + & - & - & & + & + & - & - & - & + \\
\hline 19 & BFP19 & + & + & - & - & - & + & - & + & - & + & + & - & - & + & - \\
\hline 20 & BFP20 & + & + & - & + & + & + & - & + & - & + & + & - & + & + & - \\
\hline 21 & BFP21 & + & + & - & - & - & + & - & + & - & + & + & - & - & + & - \\
\hline 22 & BFP22 & + & - & + & + & - & + & - & + & - & - & + & + & - & - & + \\
\hline 23 & BFP23 & + & - & - & - & - & + & - & + & - & + & + & - & - & - & + \\
\hline 24 & BFP24 & + & + & - & - & - & + & - & - & - & + & + & - & - & - & + \\
\hline 25 & BFP25 & + & + & - & - & - & + & - & + & - & + & + & - & - & + & - \\
\hline 26 & BFP26 & + & + & - & - & - & + & - & + & - & + & + & - & - & - & + \\
\hline 27 & BFP27 & + & + & - & - & - & + & - & + & - & + & + & - & - & + & - \\
\hline 28 & BFP28 & + & + & - & - & + & + & - & + & - & + & - & + & + & + & - \\
\hline 29 & BFP29 & + & + & - & - & + & + & - & + & - & + & + & - & + & + & - \\
\hline 30 & BFP30 & + & + & + & + & + & + & - & + & - & + & - & - & + & - & + \\
\hline 31 & BFP31 & - & + & - & - & - & - & - & + & - & - & - & - & - & + & - \\
\hline 32 & BFP32 & + & + & + & + & + & + & - & + & + & + & + & - & + & + & - \\
\hline 33 & BFP33 & + & + & + & + & + & + & - & + & + & + & + & + & + & + & - \\
\hline 34 & BFP34 & + & + & + & + & + & + & - & + & + & + & + & + & + & + & - \\
\hline 35 & BFP35 & + & + & + & + & + & + & - & + & + & + & + & + & + & + & - \\
\hline 36 & BFP36 & + & + & + & + & + & + & - & + & + & + & + & + & + & + & - \\
\hline
\end{tabular}


Int.J.Curr.Microbiol.App.Sci (2017) 6(12): 3883-3898

\begin{tabular}{|c|c|c|c|c|c|c|c|c|c|c|c|c|c|c|c|c|}
\hline 37 & BFP37 & + & + & + & + & + & + & - & + & + & + & + & - & + & + & - \\
\hline 38 & BFP38 & + & + & + & + & + & + & - & + & + & + & + & + & + & + & - \\
\hline 39 & BFP39 & + & + & + & + & - & + & - & + & - & - & - & + & - & + & - \\
\hline 40 & BFP40 & - & + & - & - & - & - & - & + & - & + & - & - & - & + & - \\
\hline 41 & BFP41 & + & + & + & + & + & + & - & + & + & + & + & + & + & - & + \\
\hline 42 & BFP42 & + & + & - & - & - & + & - & + & - & + & - & - & - & + & - \\
\hline 43 & BFP43 & + & + & - & - & + & + & - & + & - & + & + & - & + & + & - \\
\hline 44 & BFP44 & + & + & + & + & + & + & - & + & + & + & + & + & + & + & - \\
\hline 45 & BFP45 & + & + & - & - & - & + & - & + & - & + & + & - & - & + & - \\
\hline 46 & DFP46 & + & + & + & + & + & + & - & + & + & + & + & + & + & + & - \\
\hline 47 & DFP47 & + & + & + & + & + & + & - & + & + & + & + & + & + & + & - \\
\hline 48 & DFP48 & + & + & + & + & + & + & - & - & + & - & + & + & + & + & - \\
\hline 49 & DFP49 & + & + & + & + & + & + & - & + & + & + & + & - & + & + & - \\
\hline 50 & DFP50 & + & + & + & + & + & + & - & + & + & + & + & - & + & - & + \\
\hline 51 & DFP51 & + & + & + & - & + & + & - & + & + & + & + & - & + & + & - \\
\hline 52 & DFP52 & + & + & + & + & + & + & - & + & + & + & + & + & + & + & - \\
\hline 53 & DFP53 & + & + & + & + & + & + & - & + & + & + & + & - & + & + & - \\
\hline 54 & DFP54 & + & + & + & + & - & + & - & + & + & + & + & - & - & + & - \\
\hline 55 & DFP55 & + & + & + & + & + & + & - & + & + & + & + & + & + & + & - \\
\hline 56 & DFP56 & + & + & + & + & + & + & - & + & + & + & + & - & + & + & - \\
\hline 57 & DFP57 & + & + & + & + & + & + & - & + & + & + & + & + & + & - & - \\
\hline 58 & DFP58 & + & + & + & + & + & + & - & + & + & + & + & + & + & + & . \\
\hline 59 & DFP59 & + & + & + & + & + & + & - & + & + & + & + & + & + & + & 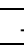 \\
\hline 60 & DFP60 & + & + & + & + & + & + & - & + & + & + & + & - & + & + & . \\
\hline 61 & DFP61 & + & - & - & - & - & + & - & + & - & - & + & - & - & + & . \\
\hline 62 & DFP62 & + & + & - & - & - & + & - & + & - & + & + & - & - & + & . \\
\hline
\end{tabular}


Table.4 Functional characterization of native fluorescent pseudomonad isolates

\begin{tabular}{|c|c|c|c|c|c|c|}
\hline $\begin{array}{l}\text { Sl. } \\
\text { No. }\end{array}$ & Isolates & $\begin{array}{c}\text { P-solubilization } \\
(\mathrm{mm})(\text { Qualitative) }\end{array}$ & $\begin{array}{c}\text { IAA } \\
(\mu \mathrm{g} / 25 \mathrm{ml})\end{array}$ & $\begin{array}{c}\text { GA } \\
(\mu \mathrm{g} / 25 \mathrm{ml})\end{array}$ & $\begin{array}{c}\text { HCN } \\
\text { production }\end{array}$ & $\begin{array}{c}\text { Siderophore } \\
\text { Production }(\mathbf{m m})\end{array}$ \\
\hline 1 & BFP1 & - & 9.28 & 0.56 & - & 8.90 \\
\hline 2 & BFP2 & - & 9.17 & 0.77 & + & 7.90 \\
\hline 3 & BFP3 & 5.31 & 9.43 & 1.43 & - & 6.00 \\
\hline 4 & BFP4 & 6.54 & 5.53 & 1.27 & - & 7.90 \\
\hline 5 & BFP5 & - & 7.15 & 0.98 & - & 8.97 \\
\hline 6 & BFP6 & - & 5.08 & 1.89 & - & 6.03 \\
\hline 7 & BFP7 & - & 3.90 & 1.37 & ++ & 10.40 \\
\hline 8 & BFP8 & 7.08 & 4.83 & 2.14 & ++ & 11.00 \\
\hline 9 & BFP9 & 5.86 & 7.86 & 1.42 & ++ & 8.97 \\
\hline 10 & BFP10 & 8.92 & 12.35 & 1.49 & + & 10.90 \\
\hline 11 & BFP11 & 7.40 & 7.43 & 2.13 & + & 10.00 \\
\hline 12 & BFP12 & - & 12.16 & 0.94 & ++ & 8.10 \\
\hline 13 & BFP13 & 11.34 & 7.41 & 1.12 & - & 8.90 \\
\hline 14 & BFP14 & 9.66 & 10.28 & 2.23 & - & 6.90 \\
\hline 15 & BFP15 & 16.67 & 8.57 & 3.15 & - & 8.97 \\
\hline 16 & BFP16 & 17.65 & 8.73 & 3.70 & + & 9.47 \\
\hline 17 & BFP17 & 10.08 & 9.64 & 1.16 & - & 12.40 \\
\hline 18 & BFP18 & 13.21 & 6.00 & 2.13 & ++ & 19.30 \\
\hline 19 & BFP19 & - & 9.08 & 3.14 & ++ & 22.20 \\
\hline 20 & BFP20 & 18.09 & 14.26 & 4.14 & ++ & 21.18 \\
\hline 21 & BFP21 & 17.18 & 8.83 & 3.17 & ++ & 14.90 \\
\hline 22 & BFP22 & 20.00 & 19.97 & 12.19 & +++ & 21.97 \\
\hline 23 & BFP23 & 19.94 & 13.14 & 4.48 & - & 10.90 \\
\hline 24 & BFP24 & 14.58 & 10.10 & 2.26 & ++ & 7.90 \\
\hline 25 & BFP25 & 18.80 & 14.57 & 2.63 & ++ & 9.90 \\
\hline 26 & BFP26 & 7.07 & 20.39 & 1.20 & +++ & 10.40 \\
\hline 27 & BFP27 & 16.00 & 18.71 & 2.22 & ++ & 8.00 \\
\hline 28 & BFP28 & 15.62 & 8.78 & 2.87 & ++ & 9.37 \\
\hline 29 & BFP29 & - & 10.82 & 3.06 & - & 13.20 \\
\hline 30 & BFP30 & 19.00 & 16.88 & 2.85 & ++ & 8.00 \\
\hline 31 & BFP31 & - & 6.00 & 4.03 & + & 21.40 \\
\hline 32 & BFP32 & 19.04 & 8.74 & 3.36 & - & 9.40 \\
\hline 33 & BFP33 & 16.74 & 17.35 & 2.52 & + & 15.70 \\
\hline 34 & BFP34 & 20.07 & 13.14 & 4.14 & + & 13.80 \\
\hline 35 & BFP35 & 17.15 & 19.34 & 2.19 & ++ & 20.10 \\
\hline 36 & BFP36 & 10.00 & 12.72 & 4.55 & ++ & 7.80 \\
\hline 37 & BFP37 & 7.31 & 11.89 & 3.19 & ++ & 9.00 \\
\hline 38 & BFP38 & 21.02 & 24.00 & 16.29 & +++ & 25.00 \\
\hline 39 & BFP39 & 18.80 & 12.18 & 9.96 & +++ & 21.17 \\
\hline 40 & BFP40 & 14.21 & 4.93 & 9.88 & - & 13.10 \\
\hline 41 & BFP41 & 14.27 & 19.21 & 7.91 & ++ & 21.10 \\
\hline 42 & BFP42 & 21.11 & 23.74 & 13.12 & +++ & 23.60 \\
\hline 43 & BFP43 & - & 11.29 & 10.16 & - & 15.30 \\
\hline 44 & BFP44 & 15.73 & 13.55 & 9.89 & ++ & 18.10 \\
\hline 45 & BFP45 & 20.12 & 19.37 & 8.97 & - & 10.10 \\
\hline 46 & DFP46 & 15.32 & 19.94 & 10.18 & ++ & 12.90 \\
\hline 47 & DFP47 & 20.96 & 22.55 & 12.85 & +++ & 23.47 \\
\hline 48 & DFP48 & 21.71 & 28.89 & 13.15 & +++ & 29.23 \\
\hline 49 & DFP49 & 17.55 & 5.88 & 8.91 & ++ & 19.83 \\
\hline 50 & DFP50 & 15.52 & 9.83 & 11.49 & ++ & 21.90 \\
\hline 51 & DFP51 & 18.65 & 12.88 & 4.91 & + & 11.30 \\
\hline 52 & DFP52 & 10.69 & 8.82 & 9.77 & + & 13.40 \\
\hline 53 & DFP53 & 16.13 & 16.77 & 0.91 & + & 21.00 \\
\hline 54 & DFP54 & 21.65 & 28.03 & 18.52 & +++ & 36.37 \\
\hline 55 & DFP55 & 19.82 & 19.00 & 10.24 & ++ & 17.87 \\
\hline 56 & DFP56 & 20.32 & 24.81 & 12.20 & +++ & 24.00 \\
\hline 57 & DFP57 & 11.00 & 19.39 & 6.89 & ++ & 22.83 \\
\hline 58 & DFP58 & 17.23 & 8.94 & 9.98 & ++ & 22.32 \\
\hline 59 & DFP59 & 20.94 & 10.00 & 10.12 & + & 14.40 \\
\hline 60 & DFP60 & 13.06 & 17.18 & 1.92 & ++ & 22.90 \\
\hline 61 & DFP61 & 20.58 & 20.06 & 10.25 & ++ & 23.10 \\
\hline \multirow[t]{3}{*}{62} & DFP62 & 20.34 & 26.41 & 12.99 & +++ & 26.70 \\
\hline & S.Em. \pm & 0.68 & 0.19 & 0.05 & - & 0.05 \\
\hline & C.D. @ $1 \%$ & 2.52 & 0.71 & 0.18 & - & \begin{tabular}{|l|l|}
0.19 \\
\end{tabular} \\
\hline
\end{tabular}

Note: (+) slight production, (++) moderate production, (+++) maximum production (-) no production 
Table.5 Antagonistic activity of fluorescent pseudomonad isolates against Sclerotium rolfsii (soil borne pathogen) under in vitro condition

\begin{tabular}{|c|c|c|c|}
\hline Sl. NO. & Isolates & Zone of inhibition $(\mathrm{cm})$ & Per cent inhibition (\%) \\
\hline 1 & BFP1 & - & - \\
\hline 2 & $\mathrm{BFP} 2$ & $3.73(11.13) *$ & $41.48(39.28)^{*}$ \\
\hline 3 & BFP3 & - & - \\
\hline 4 & BFP4 & $3.12(10.16)$ & $34.63(36.04)$ \\
\hline 5 & BFP5 & $2.85(9.72)$ & $31.70(34.53)$ \\
\hline 6 & BFP6 & $2.98(9.94)$ & $33.15(35.68)$ \\
\hline 7 & BFP7 & $2.82(9.66)$ & $31.29(34.27)$ \\
\hline 8 & BFP8 & $3.78(11.21)$ & $42.04(39.88)$ \\
\hline 9 & BFP9 & $3.00(9.97)$ & $33.33(35.41)$ \\
\hline 10 & BFP10 & - & - \\
\hline 11 & BFP11 & $2.60(9.27)$ & $28.89(32.86)$ \\
\hline 12 & BFP12 & $3.50(10.78)$ & $38.89(38.37)$ \\
\hline 13 & BFP13 & - & - \\
\hline 14 & BFP14 & - & - \\
\hline 15 & BFP15 & $2.03(8.19)$ & $22.59(29.24)$ \\
\hline 16 & BFP16 & $2.52(9.12)$ & $27.96(32.05)$ \\
\hline 17 & BFP17 & - & - \\
\hline 18 & BFP18 & - & - \\
\hline 19 & BFP19 & - & - \\
\hline 20 & BFP20 & - & - \\
\hline 21 & BFP21 & $3.03(10.02)$ & $33.70(35.50)$ \\
\hline 22 & BFP22 & $6.33(14.57)$ & $70.37(54.00)$ \\
\hline 23 & BFP23 & $3.13(10.18)$ & $34.74(36.30)$ \\
\hline 24 & BFP24 & $3.25(10.38)$ & $36.11(36.84)$ \\
\hline 25 & BFP25 & $3.32(10.49)$ & $36.85(37.06)$ \\
\hline 26 & BFP26 & $2.42(8.94)$ & $26.85(31.81)$ \\
\hline 27 & BFP27 & $2.45(9.00)$ & $27.22(32.05)$ \\
\hline 28 & BFP28 & - & - \\
\hline 29 & BFP29 & - & - \\
\hline 30 & BFP30 & 3.37 (10.57) & $37.41(37.59)$ \\
\hline 31 & BFP31 & $3.08(10.11)$ & $34.26(35.90)$ \\
\hline 32 & BFP32 & $3.32(10.49)$ & $36.85(36.84)$ \\
\hline 33 & BFP33 & $3.37(10.57)$ & $37.41(37.59)$ \\
\hline 34 & BFP34 & $3.20(10.30)$ & $35.56(36.66)$ \\
\hline 35 & BFP35 & $3.50(10.78)$ & $38.89(38.37)$ \\
\hline 36 & BFP36 & $3.35(10.54)$ & $37.22(37.41)$ \\
\hline 37 & BFP37 & $3.25(10.38)$ & $36.11(36.84)$ \\
\hline 38 & BFP38 & $4.10(11.68)$ & $45.56(41.71)$ \\
\hline 39 & BFP39 & $3.38(10.59)$ & $37.59(37.76)$ \\
\hline 40 & BFP40 & $3.12(10.16)$ & $34.63(36.04)$ \\
\hline 41 & BFP41 & $3.15(10.22)$ & $35.00(36.62)$ \\
\hline 42 & BFP42 & $4.47(12.20)$ & $49.63(43.57)$ \\
\hline 43 & BFP43 & $2.53(9.15)$ & $28.15(32.63)$ \\
\hline 44 & BFP44 & $3.32(10.49)$ & $36.89(37.41)$ \\
\hline 45 & DFP45 & $2.60(9.27)$ & $28.89(32.87)$ \\
\hline 46 & DFP46 & $3.20(10.30)$ & $35.56(36.44)$ \\
\hline 47 & DFP47 & $4.10(11.68)$ & $45.56(41.50)$ \\
\hline 48 & DFP48 & $5.30(13.30)$ & $58.89(48.17)$ \\
\hline 49 & DFP49 & 3.77 (11.19) & $41.85(39.84)$ \\
\hline 50 & DFP50 & $3.82(11.26)$ & $42.41(60.01)$ \\
\hline 51 & DFP51 & $3.42(10.65)$ & $37.96(56.97)$ \\
\hline 52 & DFP52 & $3.32(10.49)$ & $36.85(55.79)$ \\
\hline 53 & DFP53 & $3.40(10.62)$ & $37.78(56.38)$ \\
\hline 54 & DFP54 & $3.37(10.57)$ & $37.41(56.38)$ \\
\hline 55 & DFP55 & $3.25(10.38)$ & $36.11(55.26)$ \\
\hline 56 & DFP56 & $3.32(10.49)$ & 36.85 (55.79) \\
\hline 57 & DFP57 & $3.53(10.83)$ & $39.26(57.88)$ \\
\hline 58 & DFP58 & $3.58(10.91)$ & $39.81(58.01)$ \\
\hline 59 & DFP59 & $3.22(10.33)$ & $35.74(54.93)$ \\
\hline 60 & DFP60 & $3.42(10.65)$ & $37.96(56.64)$ \\
\hline 61 & DFP61 & $3.35(10.54)$ & $37.22(56.31)$ \\
\hline 62 & DFP62 & $5.48(13.54)$ & $60.93(73.52)$ \\
\hline \multirow[t]{3}{*}{63} & Control & $0.00(1.00)$ & $0.00(1.00)$ \\
\hline & S.m. \pm & 0.094 & 0.752 \\
\hline & C.D.@ $1 \%$ & 0.350 & 2.791 \\
\hline
\end{tabular}

Note: BFP isolates obtained from Belagavi soil sample and DFP isolates from Dharwad soil sample; (+) low inhibition of pathogen, (++) moderate inhibition, (+++) good inhibition and (-) no inhibition.

* Arcsine values 
Table.6 Antagonistic activity of fluorescent pseudomonad isolates against Colletotricum truncatum (foliar pathogen) under in vitro condition

\begin{tabular}{|c|c|c|c|}
\hline Sl. NO & Isolates & Zone of inhibition (cm) & Per cent inhibition \\
\hline 1 & BFP1 & - & - \\
\hline 2 & BFP2 & - & - \\
\hline 3 & BFP3 & - & - \\
\hline 4 & BFP4 & - & - \\
\hline 5 & BFP5 & - & - \\
\hline 6 & BFP6 & - & - \\
\hline 7 & BFP7 & $2.90(9.80)^{*}$ & $32.22(34.57)^{*}$ \\
\hline 8 & BFP8 & $3.73(11.14)$ & $40.81(39.69)$ \\
\hline 9 & BFP9 & $3.60(10.93)$ & $39.66(39.02)$ \\
\hline 10 & BFP10 & - & - \\
\hline 11 & BFP11 & - & - \\
\hline 12 & BFP12 & - & - \\
\hline 13 & BFP13 & - & - \\
\hline 14 & BFP14 & - & - \\
\hline 15 & BFP15 & - & - \\
\hline 16 & BFP16 & - & - \\
\hline 17 & BFP17 & - & - \\
\hline 18 & BFP18 & - & - \\
\hline 19 & BFP19 & - & - \\
\hline 20 & BFP20 & $3.92(11.41)$ & $42.70(40.79)$ \\
\hline 21 & BFP21 & - & - \\
\hline 22 & BFP22 & $6.60(14.88)$ & $74.36(59.56)$ \\
\hline 23 & BFP23 & - & - \\
\hline 24 & BFP24 & - & - \\
\hline 25 & BFP25 & - & - \\
\hline 26 & BFP26 & $2.55(9.18)$ & $28.33(32.15)$ \\
\hline 27 & BFP27 & $2.52(9.12)$ & $27.96(31.91)$ \\
\hline 28 & BFP28 & - & - \\
\hline 29 & BFP29 & - & - \\
\hline 30 & BFP30 & $2.92(9.83)$ & $32.41(34.68)$ \\
\hline 31 & BFP31 & - & - \\
\hline 32 & BFP32 & $3.30(10.46)$ & $36.67(37.25)$ \\
\hline 33 & BFP33 & $3.62(10.96)$ & $40.19(39.32)$ \\
\hline 34 & BFP34 & $3.33(10.52)$ & $37.04(37.47)$ \\
\hline 35 & BFP35 & $3.52(10.80)$ & $38.40(38.28)$ \\
\hline 36 & BFP36 & $3.98(11.51)$ & $44.40(41.77)$ \\
\hline 37 & BFP37 & $3.63(10.98)$ & $40.94(39.76)$ \\
\hline 38 & BFP38 & $3.23(10.35)$ & $36.77(37.31)$ \\
\hline 39 & BFP39 & $2.88(9.77)$ & $32.49(34.73)$ \\
\hline 40 & BFP40 & - & - \\
\hline 41 & BFP41 & $3.48(10.75)$ & $38.70(38.45)$ \\
\hline 42 & BFP42 & $5.54(13.08)$ & $63.22(52.64)$ \\
\hline 43 & BFP43 & $3.70(11.08)$ & $41.18(39.90)$ \\
\hline 44 & BFP44 & $2.20(8.52)$ & $24.44(29.62)$ \\
\hline 45 & BFP45 & $2.30(8.72)$ & $25.56(30.34)$ \\
\hline 46 & DFP46 & $3.48(10.75)$ & $38.70(38.45)$ \\
\hline 47 & DFP47 & $3.67(11.03)$ & $40.74(39.65)$ \\
\hline 48 & DFP48 & $3.37(10.57)$ & $37.41(37.69)$ \\
\hline 49 & DFP49 & $2.93(9.86)$ & $32.59(34.80)$ \\
\hline 50 & DFP50 & $3.40(10.62)$ & $37.78(37.91)$ \\
\hline 51 & DFP51 & $3.08(10.11)$ & $34.26(35.81)$ \\
\hline 52 & DFP52 & 3.75 (11.16) & $41.67(40.19)$ \\
\hline 53 & DFP53 & $3.60(10.93)$ & $40.00(39.21)$ \\
\hline 54 & DFP54 & $5.02(12.95)$ & $56.55(48.74)$ \\
\hline 55 & DFP55 & $3.57(10.88)$ & $39.63(39.00)$ \\
\hline 56 & DFP56 & $6.10(14.29)$ & $67.78(55.39)$ \\
\hline 57 & DFP57 & $3.53(10.83)$ & $39.26(38.78)$ \\
\hline 58 & DFP58 & 4.07 (11.63) & $45.57(42.44)$ \\
\hline 59 & DFP59 & $2.87(9.74)$ & $31.85(34.34)$ \\
\hline 60 & DFP60 & $4.10(11.68)$ & $45.56(42.43)$ \\
\hline 61 & DFP61 & $3.02(10.00)$ & $33.52(35.36)$ \\
\hline 62 & DFP62 & $6.35(14.59)$ & $70.84(57.30)$ \\
\hline \multirow[t]{3}{*}{63} & Control & $0.00(1.00)$ & $0.00(1.00)$ \\
\hline & S.Em. \pm & 0.110 & 0.421 \\
\hline & C.D. @ $1 \%$ & 0.420 & 1.582 \\
\hline
\end{tabular}

Note: * Arcsine formed values 
The variations in IAA production could be an inherent metabolic variability among the isolates (Leinhos and Vacek, 1994). The level of expression of IAA depended on the biosynthetic pathway, the location of genes involved and the presence of enzymes that could convert active free IAA into an inactive conjugated form (Patten and Glick, 1996).

Gibberellic acid is a class of phytohormone most commonly associated with modifying plant morphology by the extension of plant tissue, particularly the stem tissue (Salisbury, 1994). The amount of GA production by different FP isolates ranged from 0.56 to $18.52 \mu \mathrm{g} / 25 \mathrm{ml}$ of broth. The isolate DFP54 was found to produce maximum amount of GA (18.52 $\mu \mathrm{g}$ per $25 \mathrm{ml}$ broth) (Table 4). These results could be compared with those reported earlier by Lenin and Jayanti (2012), who observed the production of $\mathrm{GA}_{3}$ by isolates of Pseudomonas ranged from 6.21 to $6.80 \mu \mathrm{g}$ per $25 \mathrm{ml}$ broth. Similarly, Suneesh (2004) reported that all the 48 fluorescent Pseudomonads isolated from the moist deciduous forests produced GA in the range of 0.72 to $5.27 \mu \mathrm{g}$ per $25 \mathrm{ml}$ of broth.

The fluorescent pseudomonads have been the most widely studied group of PGPR with respect to biocontrol of soil borne plant pathogens. The increased interest in the fluorescent Pseudomonas sp. in worldwide as biocontrol agents gained momentum after the initial studies conducted at the University of California, Berkeley, during the late 1970s (Weller et al., 1988). In addition to all the beneficial traits as discussed earlier, 62 isolates were used to study their antagonistic potential against two fungal pathogens ( $S$. rolfsii and $C$. truncatum) of soybean under in vitro condition using dual plate technique (Vincent, 1947).

Among 62 isolates, 52 isolates showed antagonistic activity against one or the other phytopathogen (Table 5 and 6). Further, out of these antagonistic isolates, 51 isolates were inhibitory to $S$. rolfsii, 38 isolates were inhibitory to $C$. truncatum and 36 isolates showed efficacy against both the pathogen. Against $S$. rolfsii, the zone of inhibition varied from 2.03 to $6.33 \mathrm{~cm}$ with percent inhibition of 22.59 to 70.37 (Table 5). The maximum percent inhibition of 70.37 was observed in BFP22, which was significantly superior over all other isolates. The isolates DFP62 and DFP48 were on par with each other with percent inhibition of 60.93 and 58.89 respectively. For $C$. truncatum the zone of inhibition varied from 2.20 to $6.35 \mathrm{~cm}$ with per cent inhibition of 24.44 to 74.36 (Table 6). The isolate BFP22 exhibited maximum inhibition of about 74.36 which is followed by DFP62 (70.84), DFP56 (67.78), BFP42 (63.22) and DFP54 (56.55). These observations are in line with the earlier reports on fluorescent pseudomonads against plant pathogenic fungi like Fusarium, Rhizoctonia, Macrophomina, Pyricularia, Alternaria, Sclerotium, Colletotrichum, Pythium and Phytophthora (Mercado-Blanco et al., 2004; Bhatia et al., 2005; Ahmadzadeh et al., 2006; Rakh et al., 2011; Vishwanath et al., 2012; Manivannan et al., 2012; Prasad et al., 2013). The effectiveness of fluorescent pseudomonads against multiple pathogens is also known (Tripathi and Johri, 2002; Suneesh, 2004; Kandoliya and Vakharia, 2014; Aly et al., 2015; Arif Fouzia et al., 2016 and Megha et al., 2007b).

Important aspect of microbial antagonistic activity is best realized when it is applied for right cause. Therefore, understanding the mechanisms of antagonistic activity could be key to application of strains for specific purposes. In the present investigation, out of the 52 antagonistic isolates, 47 isolates produced HCN (Table 4). Among 47 isolates, 10 isolates were strong $(+++), 26$ isolates were moderate $(++)$ and 11 isolates were 
weak (+) HCN producer. The isolates which exhibited strong $(+++)$ HCN production (BFP22, BFP26, BFP42, DFP47, DFP54, DFP56 and DFP62) showed very good biocontrol potential against the phytopathogens tested whereas the isolates with moderate $\mathrm{HCN}$ production showed moderate biocontrol activity. Production of $\mathrm{HCN}$ is known to induce systemic resistance in plants (Wei et al., 1991). Voisard et al., (1989) reported HCN production as a mechanism of biocontrol of plant pathogens. Similarly, Ahmadzadeh and Sharifi-Tehrani (2009) detected the production of HCN by six isolates of fluorescent pseudomonads and the strains exhibited good in vitro antifungal activity against Rhizoctonia solani.

The siderophore production by antagonistic microorganisms is believed to be a mechanism of pathogen suppression. Siderophore production test using CAS agar plate has been used for rapid screening of potential beneficial bacterial isolates (Schwyn and Neiland, 1987). Bacteria with the ability to produce siderophore can enhance plant growth by increasing the availability of iron near the roots for plant uptake (Alexander and Zuberer, 1991). Although, all 52 antagonistic isolates of this study produced siderophores, the zone of clearance on CAS agar ranged from 6 to $36.37 \mathrm{~mm}$ (Table 4). Twenty one isolates produced the zone of clearance between 5 to $10 \mathrm{~mm}$, which were significantly superior to the remaining isolates. Similarly, among the isolates tested, 15 isolates recorded $10-15 \mathrm{~mm}$ zone of clearance, 3 isolates exhibited the zone of clearance between 25 to $30 \mathrm{~mm}$. The isolates DFP54 recorded highest zone of clearance of $36.36 \mathrm{~mm}$ and three isolates did not show any clearance zone.

The siderophores are usually produced by various beneficial soil microbes. Among them fluorescent pseudomonads are also involved in inhibition of $S$. rolfsii which is positively correlated $\left(\mathrm{r}=+0.336^{* *}\right)$ with production of siderophores by fluorescent pseudomonads (Indi, 2010). The concept of disease suppression by siderophores and the role of siderophores produced by fluorescent pseudomonads in plant growth promotion were explained for the first time by Kloepper et al., (1980).

This study resulted in obtaining 62 fluorescent pseudomonads from the soybean rhizosphere samples collected from Belagavi and Dharwad districts of Karnataka. Some of these isolates exhibited MPS activity, PGPR production, HCN production and siderophore production. They also showed antagonistic activity against $S$. rolfsii and $C$. truncatum under in vitro condition. Application of these microbes for diseases management and their practical use requires further investigation under field conditions.

\section{References}

Ahmadzadeh, M. and Sharifi, A., 2009. Evaluation of fluorescent pseudomonads for plant growth promotion, antifungal activity against Rhizoctonia solani on common bean and biocontrol potential. Biol. Control. 48(2): 101-107.

Alexander, D.B. and Zuberer, D. A., 1991. Use of chrome azurol $S$ reagents to evaluate siderophore production by rhizosphere bacteria. Biol. Fertil. Soil, 12: 39-45.

Aly, Neweig, Zaghlou, ElSayed, Bahloul, 2015. Evaluation of some bio-control agents against soil pathogenic fungi. Res. J. Pharma. Biol. Chem. Sci., 6(1): 439-447.

Anand, M., Naik, M.K., Ramegowda, G., Devika Rani, G.S., 2010. Biocontrol and plant growth promotion activity of indigenous Pseudomonas fluorescens isolates. J. Mycopathol. Res., 48(1): 45- 
50.

Anonymous, 1957. Manual of Microbiological Methods, McGraw Hill Book Company Inc., New York, p. 127.

Arif, F., Silini, A., Cherif, H., Ghoul, M., 2016. Plant growth promoting and antagonistic traits of indigenous fluorescent Pseudomonas spp. isolated from wheat rhizosphere and A. halimus endosphere, European Sci. J., 11(24): 1857-7881.

Ashrafuzzaman, M., Hossen, F.A., Ismail, M.R., Hoque, M.A., Islam, M.Z., Shahidullah, S. M., Meon, S., 2009. Efficiency of plant growth promoting rhizobacteria for the enhancement of rice growth. African J. Biotechnol., 8: 1247-1252.

Bakthavatchalu, S., Shivakumar, S. and Sullia, S.B., 2012. Identification of multi-trait PGPR isolates and evaluation of their potential as biocontrol agents. Acta Biologica Indica, 1(1): 61-67.

Barthalomew, J.W., Mittewar, T., 1950. A simplied bacterial stain. Stain Tech., 25: 153.

Bhatia, S., Dubey, R.C., Maheshwari, D.K., 2005. Enhancement of plant growth and suppression of collar rot of sunflower caused by Sclerotium rolfsii through fluorescent pseudomonads. Indian Phytopathol., 58(1): 17-24.

Brown, V. I., Lowbury, E.J.L., 1968. Use of an improved cetrimide agar medium and other culture methods for Pseudomonas aeruginosa. J. Clin. Path., 18: 752- 756.

Cappuccino, J.G., Sherman, N., 1992. Microbiology : A Laboratory Manual, The Benjamin/Cummins Publishing Company Inc., California.

Clarke, P.H., 1982. The metabolic versatility of Pseudomonads. Canadian J. Microbiol., 48(2): 105-130.

Haas, D., Defago G., 2005. Biological control of soil-borne pathogens by fluorescent pseudomonads. Nat. Rev. Microbiol., 3: 307-319.

Indi, D. V., 2010. Studies on plant growth promoting fluorescent pseudomonads of Uttara Kannada district of Karnataka state, P. h. D Thesis, Univ. Agric. Sci., Dharwad (India).

Kandoliya, U.K., Vakharia, D.N., 2013. Antagonistic effect of Pseudomonas fluorescens against Fusrium oxysporum f. sp. ciceri causing wilt in chickpea. Legume Res., 36(6): 569-575.

Khakipour, N., Khavazi, K., Mojallali, H., Pazira, E., Asadirahmani, H., 2008, Production of auxin hormone by fluorescent pseudomonads. AmericanEurasian J. Agric. Environ. Sci., 4(6): 687-692.

King, E.O., Ward, M.K. and Raney, D.E., 1954. Two simple media for the demonstration of pyocyanin and fluorescin. J. Lab. Clin. Med., 44: 301307.

Kloepper, J.W., Leong, J., Teintze, M., Schroth, M. N., 1980. Enhanced plant growth by siderophores produced by plant growth-promoting rhizobacteria. Nature. 286: 885-886.

Leinhos, V., Vacek, O., 1994. Biosynthesis of auxins by phosphate solubilizing rhizobacteria from wheat (Triticum aestivum) and rye (Secale cereale). Microbiol. Res., 149: 22-28.

Lenin, G., Jayanthi, M., 2012. Efficiency of plant growth promoting rhizobacteria (PGPR) on enhancement of growth, yield and nutrient content of Catharanthus roseus. Int. J. Res. Pure App. Microbiol., 2(4): 37-42.

Lugtenberg, B.J., Dekkers, L., Bloemberg, G. V., 2001. Molecular determinants of rhizosphere colonization by Pseudomonas. Annu. Rev. Phytopathol., 39: 461-490.

Manivannan, M., Ganesh, P, Suresh, R., Tharmaraj, K., Shiney, B., 2012. 
Isolation, screening, characterization and antagonism assay of PGPR isolates from rhizosphere of rice plants in Cuddalore distric. Intl. J. Pharma. Biol. Arch., 3(1): 179-185.

Martinez, V.O, Jorquera, M.A., Crowley, D.E., Gajardo, G., Mora, M.L., 2010. Mechanisms and practical considerations involved in plant growth promotion by rhizobacteria. J. Soil Sci. Pl. Nutr., 10: 293-319.

Meera, T., Balabaskar, P., 2012. Isolation and characterization of Pseudomonas fluorescens from rice fields, Intl. J. Food Agric. Vet. Sci., 2 (1): 113-120.

Megha, Y.J., Alagawadi, A.R., Krishnaraj, P.U., 2007a. Diversity of fluorescent pseudomonads isolated from the forest soils of the Western Ghats of Uttara Kannada. Curr. Sci., 93(10): 1433-1437.

Megha, Y.J., Alagawadi, A.R., Krishnaraj, P.U., 2007b. Multiple beneficial functions of fluorescent pseudomonads of Western Ghats of Uttara Kannada district. Karnataka J. Agric. Sci., 20(2): 305-309.

Mercado, J., Rodriguez, D., Hervas, A., Jimenez, R.M., 2004. Supression of Verticillium wilt in olive planting stocks by root-associated fluorescent Pseudomonas spp. Biol Cont., 30: 474486.

Mirza, M.S., Ahmad, W., Latif, F., Haurat, J., Bally, R., Normand, P., Malik, K.A., 2001. Isolation, partial characterization and the effect of plant growth promoting bacteria (PGPB) on micro propagated sugarcane in vitro. Pl. Soil., 237: 47-54.

Paramageetham, C., Babu, G., 2013. Morphological, biochemical and functional characterization of Pseudomonas fluorescence strains isolated from forest litter of Seshachalam hill range. Intl. J. Res. Pure Appl. Microbiol., 3(1): 1-3.
Patten, C.L., Glick, B.R., 1996. Bacterial biosynthesis of indole-3-acetic acid. Canadian phosphate solubilizing Pseudomonas sp. NBR14014 and their characterization. Acta Phytopathol. Entomol. Hung., 41(3): 203-212.

Prasad, J.S., Jha, M., Kumar, R.N. and Gupta, A.K., 2013, Isolation, screening and antagonism assay of Pseudomonas spp. for plant growth promoting activity and its compatibility with pesticide molecules. Bioinfolet, 10(4): 14871491.

Raaijmakers, J.M. and Weller, D.M., 1998, Natural plant protection by 2, 4diacetylphloroglucinol producing Pseudomonas sp. in take-all disease soils. Mole. Pl. Microbe Int., 11: 144152.

Rakh, R.R., 2011, Biological control of Sclerotium rolfsii, causing stem rot of groundnut by Pseudomonas spp. Recent Res. Sci. Technol., 3(3): 26-34.

Rashid, M., Khalil, S., Ayub, N., Alam, S., Latif, F., 2004. Organic acids production and phosphate solubilization by phosphate solubilizing microorganisms (PSM) under in vitro conditions. Pak. J. Biol. Sci., 7: 187196.

Sakthivel, N., Gnanamanickam, S.S., 1987. Evaluation of Pseudomonas fluorescens for suppression of sheath rot disease and enhancement of grain yields in rice (Oryza sativa L.). Appl. Environ. Microbiol. 47: 2056-2059.

Salisbury, F.B., 1994. The role of plant hormones in plant environment interactions. (Eds) Wilkinson, R. E., Marcel Dekker, New York, USA, pp. 39-81.

Saravanakumar, D., Muthumeena, K., Lavanya, N. Suresh, S. Rajendran, L., Raguchander, T. and Samiyappan, R., 2007b. Pseudomonas-induced defence molecules in rice plants against leaf 
folder (Cnaphalocrocis medinalis) pest. Pest Managt. Sci., 63(7) : 714-721.

Sarwar, M. and Kremer, R. J., 1992. Determination of bacterially derived auxins using a microplate method. Let. Appl. Microbiol., 20: 282-285.

Scarpellini, M., Franzetti, L., Galli, A., 2004. Development of PCR assay to identify Pseudomonas fluorescens and its biotype. FEMS Microbiol. Lett., 236 :257-260.

Schwyn, B. Neilands, J.B., 1987. Universal chemical assay for the detection and determination of siderophores. Analytical Biochem., 160: 47-56.

Singh A.K., Pandey M.B., Singh U.P. 2007. Antifungal activity of an alkaloid allosecurinine against some fungi. Mycobiol. 35: 62-64.

Suman, B., Gopal, A.V., Reddy, R.S., Triveni, S., 2016. Isolation and characterization of Pseudomonas fluorescens in the rice rhizospheric soils of Rangareddy district in Telangana state. Int. J. Microbiol. Rese. Rev. 5 (1): 64-169.

Suneesh, K. 2004. Biodiversity of fluorescent Pseudomonads in soils of moist deciduous forests of Western Ghats of Uttar Kannada district. M. Sc. (Agri). Thesis, Uni. Agric. Sci, Dharwad.
Tripathi, M. and Johri, B.N., 2002. In vitro antagonistic potential of fluorescent pseudomonads and control of sheath blight of maize caused by Rhizoctonia solani. Indian J. Microbiol., 42(3): 207214.

Vincent, J.M., 1927, Distortion of fungal hyphae in the presence of certain inhibitor. Nature, 159: 800.

Vishwanath, P., Shankar, S., Suvarna, V.C., Jayasheela, 2012, Biological control of collar rot of sunflower using rhizobacteria. Intl. J. Pl. Protect. 5(2): 391-393.

Voisard, C., Keel, O., Haas, P., Defago, G., 1989. Cyanide production by Pseudomonas fluorescens helps to suppress black root rot of tobacco under gnotobiotic condition. European Microbiol. J., 8: 351-358.

Wei, G., Kloepper, J.W., Tuzun, S., 1991. Induction of systemic resistance of cucumber to Colletotrichum orbiculare by selected strains of plant growth promoting rhizobacteria. Phytopathol., 81: 1508-1512.

Weller, D.M., Howke, W.J. and Cook, R.J., 1988. Biological control of soil borne plant pathogens in the rhizosphere with bacteria. Phytopathol., 38: 1094.

\section{How to cite this article:}

Priyanka, Geeta Goudar, P. Jones Nirmal Nath and Patil, P.V. 2017. Isolation, Characterization and Antagonistic Activity of Fluorescent Pseudomonads. Int.J.Curr.Microbiol.App.Sci. 6(12): 3883-3898. doi: https://doi.org/10.20546/ijcmas.2017.612.449 